\title{
A LOWER BOUND ON THE GROWTH EXPONENT FOR LOOP-ERASED RANDOM WALK IN TWO DIMENSIONS
}

\author{
GREGORY F. LAWLER ${ }^{1}$
}

\begin{abstract}
The growth exponent $\alpha$ for loop-erased or Laplacian random walk on the integer lattice is defined by saying that the expected time to reach the sphere of radius $n$ is of order $n^{\alpha}$. We prove that in two dimensions, the growth exponent is strictly greater than one. The proof uses a known estimate on the third moment of the escape probability and an improvement on the discrete Beurling projection theorem.

Résumé. L'exposant de croissance $\alpha$ pour la marche aléatoire à boucles effacées ou "laplacienne" sur le réseau $Z^{d}$ est défini de la manière suivante : le nombre moyen de pas au moment où la marche issue de l'origine atteint la sphère de rayon $n$ est d'ordre $n^{\alpha}$ lorsque $n$ tend vers l'infini. Nous montrons que lorsque $d=2$, l'exposant de croissance est strictement supérieur à 1 . La preuve utilise une estimation connue concernant le moment d'ordre trois de la probabilité de fuite, ainsi qu'un raffinement de la version discrétisée du théorème de projection de Beurling.
\end{abstract}

AMS Subject Classification. 60J15.

Received April 10, 1998. Revised September 21, 1998.

\section{INTRODUCTION}

Loop-erased or Laplacian random walk (LERW) on the integer lattice $\mathbb{Z}^{d}, d \geq 2$, is a non Markovian, nearest neighbor, self-avoiding process. This process was originally studied because it is a nontrivial process that is self-avoiding, although there is very strong numerical and nonrigorous analytic evidence $[4,6,9,16]$ to believe that it is not in the same universality class as the usual self-avoiding walk. There has been a recent interest in the LERW because of the connection between loop-erased walk and uniform spanning trees [16-18].

There are a number of ways to define the loop-erased walk. If $d \geq 3$, one can take an infinite simple random walk and erase the loops chronologically to produce a self-avoiding path. This is well-defined for $d \geq 3$ since the simple random walk is transient. A somewhat modified definition is needed in two dimensions, and since this paper will concentrate on $d=2$ we will give this definition, which also works for higher dimensions. If $\omega=[\omega(0), \ldots, \omega(n)]$ is any nearest neighbor path in $\mathbb{Z}^{d}$, we define the loop-erased path $L(\omega)$ as follows. Let

$$
s_{0}=\sup \{k \leq n: \omega(k)=\omega(0)\},
$$

and if $s_{j}<n$,

$$
s_{j+1}=\sup \left\{k \leq n: \omega(k)=\omega\left(s_{j}+1\right)\right\} .
$$

Keywords and phrases: loop-erased walk, Beurling projection theorem

${ }^{1}$ Department of Mathematics, Box 90320, Duke University Durham, NC 27708-0320, USA; e-mail: jose@math.duke.edu 
If $l$ is the smallest index so that $s_{l}=n$, the loop-erased path is given by

$$
L(\omega)=L[\omega(0), \ldots, \omega(n)]=\left[\omega\left(s_{0}\right), \ldots, \omega\left(s_{l}\right)\right] .
$$

Note the $L(\omega)$ is a self-avoiding, nearest neighbor path whose initial and end points are the same as those of $\omega$.

Let $S(k)$ denote a simple random walk in $\mathbb{Z}^{d}, d \geq 2$, starting at the origin. Let

$$
C_{m}=\left\{x \in \mathbb{Z}^{d}:|x|<m\right\},
$$

with boundary

$$
\partial C_{m}=\left\{x \in \mathbb{Z}^{d} \backslash C_{m}:|y-x|=1 \text { for some } y \in C_{m}\right\} .
$$

Let

$$
\sigma_{m}=\inf \left\{k: S(k) \in \partial C_{m}\right\}
$$

Let $\Lambda_{n}$ be the set of nearest neighbor, self-avoiding paths $\omega=[0=\omega(0), \ldots, \omega(l)]$ of any length $l$ such that $\omega(k) \in C_{n}, k<l$, and $\omega(l) \in \partial C_{n}$. If $n \leq m$, there is a measure on $\Lambda_{n}, \mu_{n . m}$ obtained by considering the unique initial segment of $L\left(S\left[0, \sigma_{m}\right]\right)$ that is in $\Lambda_{n}$. We define the measure $\mu_{n}$ as the limit as $m$ tends to infinity of the measures $\mu_{n, m}$. It can be shown ([9], Chapter 7$)$ that the limit exists, and the measures $\left\{\mu_{n}\right\}$ are consistent. Hence this gives a measure on infinite self-avoiding paths. This measure is the same as the measure produced by a process $\hat{S}(n)$ with the following non Markovian transition probabilities ([9], Chapter 7). If $x \in \mathbb{Z}^{d}$, and $\omega=[\omega(0), \ldots, \omega(k)]$ is a finite self-avoiding path, let

$$
f_{m}(x, \omega)=\mathbf{P}^{x}\left\{S\left[0, \sigma_{m}\right] \cap \omega=\emptyset\right\}
$$

(here we make the natural identification of a path with its range). Then if $|x-\omega(k)|=1$,

$$
\mathbf{P}\{\hat{S}(k+1)=x \mid[\hat{S}(0), \ldots, \hat{S}(k)]=\omega\}=\lim _{m \rightarrow \infty} \frac{f_{m}(x, \omega)}{\sum_{|y-\omega(k)|=1} f_{m}(y, \omega)} .
$$

Also from the discrete Harnack principle ( [9], Th. 1.7.6), there exists a constant $c$ such that if $\omega \subset C^{m / 2}$,

$$
c^{-1} \frac{f_{m}(x, \omega)}{\sum_{|y-\omega(k)|=1} f_{m}(y, \omega)} \leq \mathbf{P}\{\hat{S}(k+1)=x \mid[\hat{S}(0), \ldots, \hat{S}(k)]=\omega\} \leq c \frac{f_{m}(x, \omega)}{\sum_{|y-\omega(k)|=1} f_{m}(y, \omega)} .
$$

In fact, it can be shown (see [13]) that if $n \leq m / 2, \omega \in \Lambda_{n}$,

$$
c^{-1} \mu_{n} \leq \mu_{n, m} \leq c \mu_{n} .
$$

We are interested in the exponent that measures the rate of growth of $\hat{S}$. This exponent is often phrased in terms of the mean squared distance $\mathbf{E}\left[|\hat{S}(n)|^{2}\right]$. We will use a different, but presumably equivalent, formulation. (While we have not shown rigorously that these formulations are equivalent, we certainly expect them to be.) Let

$$
\hat{\sigma}_{n}=\inf \left\{k: \hat{S}(k) \in \partial C_{n}\right\} .
$$

We would like to define the exponent $\alpha=\alpha_{d}$ by the relation

$$
\mathbf{E}\left[\hat{\sigma}_{n}\right] \approx n^{\alpha},
$$


where $\approx$ indicates that the logarithms of boths sides are asymptotics. Intuitively we say that the paths of the loop-erased walk have fractal dimension $\alpha$. If $d \geq 4$, it is known $[9,11]$ that $\alpha=2$, with logarithmic corrections in four dimensions. Since we have no proof that $\alpha$ exists for $d=2,3$, to be precise we should define $\underline{\alpha}$ and $\bar{\alpha}$ to be the liminf and limsup, respectively, of

$$
\frac{\log \mathbf{E}\left[\hat{\sigma}_{n}\right]}{\log n}
$$

By slight abuse of notation we will write $\alpha \geq s$ to mean $\underline{\alpha} \geq s$ and $\alpha \leq s$ for $\bar{\alpha} \leq s$. For $d=2,3$, there is an upper bound [9]

$$
\alpha_{d} \leq \frac{d+2}{3},
$$

but numerical simulations [6] indicate that this bound is not sharp. The right hand side is exactly the Flory predictions for the corresponding quantity for the usual self-avoiding walk (see [15] for more details about self-avoiding walks and the Flory predictions). This prediction is still expected to be correct for the usual selfavoiding walk in two dimensions, but is expected to be slightly lower than the actual value in three dimensions. The conjectures imply that the LERW goes to infinity faster than the usual simple random walk. By comparison to uniform spanning trees and the Potts model [16], nonrigorous conformal field theory $[4,16]$ has been used to conjecture that

$$
\alpha_{2}=\frac{5}{4}
$$

There is no reason to believe that $\alpha_{3}$ is a nice rational number; numerical simulations do suggest that $\alpha_{3}<5 / 3$. The rigorous inequality $\alpha \geq 1$ is immediate. By comparison to the intersection exponent in three dimensions [3], it can be proved that $\alpha_{3}>1$, but no such inequality has been shown in two dimensions. The purpose of this paper is to prove $^{1}$

$$
\alpha_{2}>1
$$

Theorem 1.1. There exist positive constants $c, \epsilon$ such that if $d=2$, for all $n$,

$$
\mathbf{E}\left[\hat{\sigma}_{n}\right] \geq c n^{1+\epsilon} .
$$

Throughout this paper we use $c, c_{1}, c_{2}$ for positive constants whose value may change from line to line. For the remainder of the paper we will assume that $d=2$. By (1.1), to prove this estimate for a given $n$ it suffices to consider the simple random walk up to time $\sigma_{2 n}$; erase loops from this path; and give an appropriate lower bound on the number of points before time $\sigma_{n}$ that are not erased. Let $V(j, n)$ be the event that $j \leq \sigma_{n}$ and that $S(j)$ is not erased in producing $L\left(S\left[0, \sigma_{2 n}\right]\right)$. More precisely,

$$
V(j, n)=\left\{j \leq \sigma_{n} ; L(S[0, j]) \cap S\left[j+1, \sigma_{2 n}\right]=\emptyset\right\} .
$$

Note that if $\hat{\sigma}_{n, 2 n}$ is the analogue of $\hat{\sigma}_{n}$ for walks stopped upon reaching $\partial C_{2 n}$,

$$
\hat{\sigma}_{n, 2 n}=\sum_{j=0}^{\infty} I[V(j, n)]
$$

\footnotetext{
${ }^{1}$ After this paper was completed, Kenyon [7] proved that $\alpha_{2}=5 / 4$.
} 
where $I$ denotes the indicator function. Hence in order to prove the theorem it suffices to prove that there exist $c, \epsilon$ such that for $n^{2} \leq j \leq 2 n^{2}$,

$$
\mathbf{P}[V(j, n)] \geq c n^{-1+\epsilon}
$$

The distribution of $L(S[0, j])$ is the same whether we consider $S(0)$ or $S(j)$ as the origin, i.e. "reverse" looperased walk has the same distribution as LERW ( [9], Lem. 7.2.1). To prove (1.3) it suffices to prove the following estimate. Let $S^{1}, S^{2}$ be independent simple random walks starting at the origin with corresponding stopping times $\sigma_{n}^{1}, \sigma_{n}^{2}$. Then for some $c, \epsilon$,

$$
\mathbf{P}\left\{L\left(S^{1}\left[0, \sigma_{n}^{1}\right]\right) \cap S^{2}\left(0, \sigma_{n}^{2}\right]=\emptyset\right\} \geq c n^{-1+\epsilon}
$$

That is, the probability that a simple walk and a loop-erased walk get to distance $n$ without intersecting is greater than $c n^{-1+\epsilon}$. This is the main estimate of this paper.

Assume for ease that $S^{i}$ is defined on the probability space $\left(\Omega_{i}, \mathbf{P}_{i}\right)$ and let $\mathbf{E}_{i}$ denote expectations with respect to $\mathbf{P}_{i}$. Define the $\Omega_{1}$ random variable

$$
X_{n}=\mathbf{P}_{2}\left\{S^{2}\left(0, \sigma_{n}^{2}\right] \cap L\left(S^{1}\left[0, \sigma_{n}^{1}\right]\right)=\emptyset\right\}
$$

It can be shown that,

$$
\mathbf{E}\left[X_{n}^{3}\right] \asymp n^{-2}
$$

where $\asymp$ means that both sides are comparable, i.e., bounded by a constant times a multiple of the other side. The upper bound for $\mathbf{E}\left[X_{n}^{3}\right]$, at least up to logarithmic corrections, can be found in [9]; this and the inequality $\mathbf{E}\left[X_{n}^{3}\right] \geq\left[\mathbf{E}\left(X_{n}\right)\right]^{3}$ give the estimate (1.2). The lower bound is what is needed in this paper; the proof will appear in [5] as well as the analogous result for three dimensions, but since we need it, we will give a proof of

$$
\mathbf{E}\left[X_{n}^{3}\right] \geq c n^{-2}
$$

in this paper.

The discrete Beurling projection theorem [8] states that if $\omega$ is any simple random walk path in $\mathbb{Z}^{2}$ connecting the origin with $\partial C_{n}$, then

$$
\mathbf{P}_{2}\left\{S^{2}\left(0, \sigma_{n}^{2}\right] \cap \omega=\emptyset\right\} \leq c n^{-1 / 2}
$$

The probability on the left is maximized (at least up to a multiplicative constant) when $\omega$ is a half line, in which case this probability is also greater than $c_{1} n^{-1 / 2}$. This theorem implies that $X_{n} \leq c n^{-1 / 2}$, and hence

$$
\mathbf{E}\left[X_{n}\right] \geq \operatorname{cn} \mathbf{E}\left[X_{n}^{3}\right]
$$

This is not good enough to get (1.4); in fact this only allows us to conclude the trivial inequality $\alpha \geq 1$. However, a typical loop-erased walk is more crooked than a straight line. The main technical tool in this paper is an upper bound on the escape probability for walks in terms of a particular quantity that measures crookedness. In particular, we will show that there is a $c$ and a $\delta>0$ such that for all large $n$

$$
\mathbf{P}_{1}\left\{X_{n} \geq c n^{-1 / 2-\delta}\right\} \leq n^{-3}
$$

In particular,

$$
\mathbf{E}_{1}\left[X_{n}^{3} ; X_{n} \leq c n^{-1 / 2-\delta}\right] \geq c_{1} n^{-2}
$$


and hence,

$$
\mathbf{E}_{1}\left[X_{n}\right] \geq c n^{-1+2 \delta}
$$

This gives (1.4).

The improvement on the discrete Beurling projection theorem is of independent interest. Let $C^{m}=C_{e^{m}}$ and suppose $\omega$ is a self-avoiding random walk path starting at the origin ending at $C^{m}$. Let

$$
\eta_{k}=\inf \left\{t: \omega(t) \in \partial C^{k}\right\}
$$

For each $0<\delta<\pi / 2$, let

$$
Z_{\delta, m}=Z_{\delta, m}(\omega)=\sum_{k=0}^{m-1} I\left\{\left|\arg \left(\omega\left(\eta_{k+1}\right)\right)-\arg \left(\omega\left(\eta_{k}\right)\right)\right| \geq \delta\right\}
$$

We will prove the following. If $S$ is a simple random walk, let

$$
\tau_{m}=\sigma_{e^{m}}=\inf \left\{t: S(t) \in \partial C^{m}\right\}
$$

Proposition 1.2. For every $\delta, a>0$, there exist $c<\infty, \beta>1 / 2$, such that if $\omega$ is any self-avoiding random walk path connecting 0 with $\partial C^{m}$ with

$$
Z_{\delta, m} \geq a m
$$

then

$$
\mathbf{P}\left\{S\left(0, \tau_{m}\right] \cap \omega=\emptyset\right\} \leq c e^{-\beta m} .
$$

We first prove a corresponding proposition for Brownian motion using the relationship between harmonic measure and extremal distance. This method has been used before, see, e.g., [20], to give probabilistic proofs of the Beurling projection theorem. The result for random walk is obtained using a strong approximation derived from the Skorohod embedding.

The outline of the paper is as follows. In Section 2 we derive the estimate on $\mathbf{E}\left[X_{n}^{3}\right]$; the argument is similar to that in [11] where a corresponding result was proved for $d=4$. The next section gives exponential estimates on the probability that the loop-erased walk is very straight. The goal of Section 4 is to prove the analogue of Proposition 1.2 for Brownian motion. The derivation uses a relationship between escape probabilities in two dimensions and a quantity, extremal distance, of a domain. The last two sections derive the result for random walk. Section 5 reviews the necessary facts about the strong approximation and then Section 6 uses this to obtain the result. A similar idea with added complications has been used in [12,14] to estimate probabilities that paths of random walks do not intersect each other with the corresponding probabilities for Brownian motion.

This research is partially supported by the National Science Foundation. I would like to thank the referee for useful comments.

\section{THIRD MOMENT}

In this section we derive (1.6). If $A \subset \mathbb{Z}^{2}$, let

$$
\operatorname{Es}_{n}(A)=\mathbf{P}\left\{S\left(0, \sigma_{n}\right] \cap A=\emptyset\right\}
$$

Lemma 2.1. Suppose $A \subset \mathbb{Z}^{2}$ contains at least one nearest neighbor of the origin. Then for all $n \geq 1$,

$$
\operatorname{Es}_{n}(A \backslash\{0\}) \leq 4 \operatorname{Es}_{n}(A) .
$$


Proof. Let $A^{\prime}=A \backslash\{0\}$, and

$$
\eta_{n}=\inf \left\{t>0: S(t) \in \partial C_{n} \cup\{0\}\right\} .
$$

Since $A^{\prime}$ contains a nearest neighbor of the origin,

$$
\mathbf{P}\left\{S\left(0, \eta_{n}\right] \cap A^{\prime} \neq \emptyset\right\} \geq \frac{1}{4} .
$$

Let $Z_{n}$ be the number of visits to 0 by $S$ before leaving $C_{n}$,

$$
Z_{n}=\sum_{t=0}^{\sigma_{n}} I\{S(t)=0\} .
$$

Then by iterating above

$$
\mathbf{P}\left\{Z_{n}=k+1, S\left(0, \sigma_{n}\right] \cap A^{\prime}=\emptyset\right\} \leq\left(\frac{3}{4}\right)^{k} \mathbf{P}\left\{S\left(0, \sigma_{n}\right] \cap A=\emptyset\right\} .
$$

Summing over $k$ gives the lemma.

If $S$ is a simple random walk starting at the origin, define the random variable

$$
X_{n}=\operatorname{Es}_{n}\left(L\left(S\left[0, \sigma_{n}\right]\right)\right) .
$$

It is the goal of this section to show that

$$
\mathbf{E}\left[X_{n}^{3}\right] \geq c n^{-2}
$$

for some constant $c$. By the lemma, it suffices to show that

$$
\mathbf{E}\left[\left(X_{n}^{\prime}\right)^{3}\right] \geq c n^{-2}
$$

where

$$
X_{n}^{\prime}=\operatorname{Es}_{n}\left(L\left(S\left[0, \sigma_{n}\right]\right) \backslash\{0\}\right) .
$$

Let $S^{1}, \ldots, S^{4}$ be independent simple random walks starting at the origin with stopping times $\sigma_{n}^{1}, \ldots, \sigma_{n}^{4}$. Let

$$
\Theta_{n}=S^{2}\left(0, \sigma_{n}^{2}\right] \cup S^{3}\left(0, \sigma_{n}^{3}\right] \cup S^{4}\left(0, \sigma_{n}^{4}\right]
$$

Then, by independence,

$$
\mathbf{E}\left[\left(X_{n}^{\prime}\right)^{3}\right]=\mathbf{P}\left\{\Theta_{n} \cap\left[L\left(S\left[0, \sigma_{n}^{1}\right]\right) \backslash\{0\}\right]=\emptyset\right\}
$$

Let $\left[S^{i}(t)\right]_{1},\left[S^{i}(t)\right]_{2}$ denote the first and second components of the random walk $S^{i}$. Let $\mathcal{A}_{n}^{1}, \ldots, \mathcal{A}_{n}^{4}$, denote the rectangles,

$$
\mathcal{A}_{n}^{1}=\left\{(x, y) \in \mathbb{Z}^{2}:-2 n \leq x \leq \frac{n}{4} ;-\frac{n}{4} \leq y \leq \frac{n}{4}\right\}
$$




$$
\begin{aligned}
& \mathcal{A}_{n}^{2}=\left\{(x, y) \in \mathbb{Z}^{2}:-\frac{n}{4} \leq x \leq 2 n ;-\frac{n}{4} \leq y \leq \frac{n}{4}\right\}, \\
& \mathcal{A}_{n}^{3}=\left\{(x, y) \in \mathbb{Z}^{2}:-\frac{n}{4} \leq x \leq \frac{n}{4} ;-2 n \leq y \leq \frac{n}{4}\right\}, \\
& \mathcal{A}_{n}^{4}=\left\{(x, y) \in \mathbb{Z}^{2}:-\frac{n}{4} \leq x \leq \frac{n}{4} ;-\frac{n}{4} \leq y \leq 2 n\right\} .
\end{aligned}
$$

For $n^{2} \leq t_{1}, t_{2}, t_{3}, t_{4} \leq 2 n^{2}$, let $U_{n}\left(t_{1}, \ldots, t_{4}\right)$ be the event that the following holds:

$$
\begin{array}{ll}
S^{i}\left[0, t_{i}\right] \subset \mathcal{A}_{n}^{i}, & i=1,2,3,4 \\
\left|S^{i}(t)\right| \geq n, \quad n^{2} \leq t \leq t_{i}, & i=1,2,3,4
\end{array}
$$

Let $V_{n}\left(t_{1}, \ldots, t_{4}\right)$ be the event

$$
V_{n}=\left\{\Theta_{n} \cap\left[L\left(S\left[0, \sigma_{n}^{1}\right]\right) \backslash\{0\}\right]=\emptyset\right\}
$$

We will show that

$$
\left.\sum_{n^{2} \leq t_{1}, t_{2} \leq 2 n^{2}} \mathbf{P}\left[U_{n}\left(t_{1}, t_{2}, n^{2}, n^{2}\right)\right] \cap V_{n}\left(t_{1}, t_{2}, n^{2}, n^{2}\right)\right] \geq c n^{2} .
$$

By the obvious monotonicity this implies

$$
\mathbf{P}\left[U_{n}\left(n^{2}, n^{2}, n^{2}, n^{2}\right) \cap V_{n}\left(n^{2}, n^{2}, n^{2}, n^{2}\right)\right] \geq c n^{-2} .
$$

From this (2.1) follows easily.

Let $S^{5}$ be a random walk starting at the origin and let $G_{n}$ be the event that the following four conditions hold:

$$
\begin{aligned}
{\left[S^{5}(t)\right]_{2} } & \leq \frac{n}{8}, \quad 0 \leq t \leq 4 n^{2}, \\
\frac{5 n}{4} & \leq\left[S^{5}(t)\right]_{1} \leq \frac{7 n}{4}, \\
\frac{5}{4} n^{2} & \leq t \leq \frac{7}{4} n^{2} \\
{\left[S^{5}(t)\right]_{1} } & \leq n, \quad 0 \leq t \leq n^{2}, \\
{\left[S^{5}(t)\right]_{1} } & \geq 3 n, \quad 2 n^{2} \leq t \leq 4 n^{2} .
\end{aligned}
$$

It is easy to see from the invariance principle that there is a constant $c$ such that

$$
\mathbf{P}\left[G_{n}\right] \geq c .
$$

Now start another random walk $S^{6}$, independent of $S^{5}$, starting at a point $x \in \mathbb{Z}^{2}$, chosen from the discrete ball of radius $n / 16$ around $(3 n / 2,-2 n)$. Note that the number of points in this ball is comparable to $n^{2}$. Let $H_{n}$ be the event that the following conditions hold:

$$
\frac{11}{8} n \leq\left[S^{6}(t)\right]_{1} \leq \frac{13}{8} n, \quad 0 \leq t \leq 4 n^{2},
$$




$$
\begin{gathered}
-\frac{n}{4} \leq\left[S^{6}(t)\right]_{2} \leq \frac{n}{4}, \quad \frac{5}{4} n^{2} \leq t \leq \frac{7}{4} n^{2} \\
{\left[S^{6}(t)\right]_{2} \leq-\frac{3 n}{2}, \quad 0 \leq t \leq n^{2},} \\
{\left[S^{6}(t)\right]_{2} \geq \frac{3 n}{2}, \quad 2 n^{2} \leq t \leq 4 n^{2} .}
\end{gathered}
$$

Again, by the invariance principle, there is a constant $c$, independent of the starting point, such that if $x$ is in the ball of radius $n / 16$ around $(3 n / 2,-2 n)$,

$$
\mathbf{P}^{x}\left[H_{n}\right] \geq c,
$$

and hence for such $x$ by independence $\mathbf{P}^{x}\left(G_{n} \cap H_{n}\right) \geq c$ (here the $x$ refers to the starting point of the walk $\left.H_{n}\right)$. By summing, we get

$$
\sum_{x} \mathbf{P}^{x}\left(G_{n} \cap H_{n}\right) \geq c n^{2}
$$

Consider the loop-erased path $L\left(S^{5}\left[0,4 n^{2}\right]\right)$. On the event $G_{n} \cap H_{n}$ we can see from geometric considerations that the paths $L\left(S^{5}\left[0,4 n^{2}\right]\right)$ and $S^{6}\left[0,4 n^{2}\right]$ must intersect, and the points of intersection must occur in the set

$$
\left\{(x, y): \frac{11}{8} n \leq x \leq \frac{13}{8} n,-\frac{1}{8} n \leq y \leq \frac{1}{8} n\right\} .
$$

Let $\rho^{1}=\rho_{n}^{1}$ be the smallest time $\mathrm{t}$ such that the point $S^{5}(t)$ is included in the loop-erased path and $S^{6}\left[0,4 n^{2}\right]$. More precisely, $\rho^{1}$ is defined by the conditions:

$$
L\left(S^{5}\left[0, \rho^{1}\right]\right) \cap S^{5}\left[\rho^{1}+1,4 n^{2}\right]=\emptyset, S^{5}\left(\rho^{1}\right) \in S^{6}\left[0,4 n^{2}\right] .
$$

Note that on $G_{n} \cap H_{n}, n^{2} \leq \rho^{1} \leq 2 n^{2}$. Let

$$
\rho^{2}=\inf \left\{t: S^{6}(t)=S^{5}\left(\rho^{1}\right)\right\} .
$$

Again, on $G_{n} \cap H_{n}, n^{2} \leq \rho^{2} \leq 2 n^{2}$. Hence

$$
\sum_{x} \sum_{n^{2} \leq t_{1}, t_{2}, \leq 2 n^{2}} \mathbf{P}^{x}\left[G_{n} \cap H_{n} ; \rho_{1}=t_{1} ; \rho_{2}=t_{2}\right] \geq c n^{2},
$$

where the outer sum is over all $x$ in the ball of radius $n / 16$ about $(3 n / 2,-2 n)$.

We define the random walks $S^{1}, \ldots, S^{4}$ in terms of $S^{5}, S^{6}$. Let

$$
\begin{aligned}
& S^{1}(t)=S^{5}\left(\rho^{1}-t\right)-S^{5}\left(\rho^{1}\right), \quad t=0,1, \ldots, \rho^{1}, \\
& S^{2}(t)=S^{6}\left(\rho^{2}-t\right)-S^{6}\left(\rho^{2}\right), \quad t=0,1, \ldots, \rho^{2}, \\
& S^{3}(t)=S^{5}\left(\rho^{1}+t\right)-S^{5}\left(\rho^{1}\right), \quad t=0,1,2, \ldots, n^{2}, \\
& S^{4}(t)=S^{6}\left(\rho^{2}+t\right)-S^{6}\left(\rho^{2}\right), \quad t=0,1,2, \ldots, n^{2} .
\end{aligned}
$$

Then in terms of these random walks, we can see that

$$
\sum_{x} \mathbf{P}^{x}\left[G_{n} \cap H_{n} ; \rho_{1}=t_{1} ; \rho_{2}=t_{2}\right] \geq P\left[V\left(t_{1}, t_{2}, n^{2}, n^{2}\right)\right] .
$$


This combined with (2.3) gives (2.2) and hence proves (2.1).

We note that a similar argument will work in $d=3$. One complication arises in that one must show that the loop-erasure of $S^{5}$ and the random walk $S^{6}$ intersect with positive probability independent of $n$. This is not an issue in two dimensions since the continuous curves must cross. For a proof of

$$
\mathbf{E}\left[X_{n}^{3}\right] \geq c n^{-1}
$$

in three dimensions, see [5]. This proof can also be adapted easily to prove the upper bound

$$
\mathbf{E}\left[X_{n}^{3}\right] \leq c n^{d-4} .
$$

Here, one essentially needs only the trivial estimate that the probability that $S^{5}$ and $S^{6}$ intersect is of order one, although a few other minor technicalities arise.

\section{CROOKEDNESS OF LOOP-ERASED WALK}

Let $S$ be a simple random walk in $\mathbb{Z}^{2}$ and

$$
\tau_{n}=\sigma_{e^{n}}=\inf \left\{t: S(t) \in \partial C^{n}\right\} .
$$

Let $\hat{S}_{n}(j)$ denote the walk obtained by erasing loops from $S\left[0, \tau_{n}\right]$. This gives a measure on self-avoiding paths starting at the origin and ending upon reaching $\partial C^{n}$. If $k \leq n$, let

$$
\hat{\tau}_{k, n}=\inf \left\{t: \hat{S}_{n}(t) \in \partial C^{k}\right\}, \quad \hat{\tau}_{k}=\inf \left\{t: \hat{S}(t) \in \partial C^{k}\right\} .
$$

Let

$$
\begin{aligned}
& \hat{W}_{n, \delta}=\sum_{k=1}^{n} I\left\{\left|\arg \left[\hat{S}_{n}\left(\hat{\tau}_{k, n}\right)\right]-\arg \left[\hat{S}_{n}\left(\hat{\tau}_{k-1, n}\right)\right]\right| \leq \delta\right\}, \\
& \tilde{W}_{n, \delta}=\sum_{k=1}^{n} I\left\{\left|\arg \left[\hat{S}\left(\hat{\tau}_{k}\right)\right]-\arg \left[\hat{S}\left(\hat{\tau}_{k-1}\right)\right]\right| \leq \delta\right\},
\end{aligned}
$$

Proposition 3.1. For every $M<\infty$ and every $\epsilon>0$, there exist $\delta>0$ and $c<\infty$ such that for all $n$,

$$
\begin{aligned}
& \mathbf{P}\left\{\hat{W}_{n, \delta} \geq \epsilon n\right\} \leq c e^{-M n} . \\
& \mathbf{P}\left\{\tilde{W}_{n, \delta} \geq \epsilon n\right\} \leq c e^{-M n} .
\end{aligned}
$$

The second inequality follows immediately from the first and (1.1), so we will only prove the first. We will actually prove the following stronger proposition. Standard large deviation estimates for binomial random variables (see, e.g. [2], Th. 9.3) can be used to derive Proposition 3.1 from Proposition 3.2.

Proposition 3.2. For every $\epsilon>0$, there exist a $\delta>0$ and $a K<\infty$, such that for any $K \leq k \leq n-1$,

$$
\mathbf{P}\left\{\left|\arg \left[\hat{S}_{n}\left(\hat{\tau}_{k, n}\right)\right]-\arg \left[\hat{S}_{n}\left(\hat{\tau}_{k-1, n}\right)\right]\right| \leq \delta \mid \hat{S}_{n}(t), t=0, \ldots, \hat{\tau}_{k-1, n}\right\} \leq \epsilon .
$$

Fix $k \leq n-1$ and suppose we know

$$
\left[\hat{S}_{n}(0), \hat{S}_{n}(1), \ldots, \hat{S}_{n}\left(\hat{\tau}_{k-1, n}\right)\right]
$$


Analysis of the loop-erasing procedure shows that to determine the distribution

$$
\left\{\hat{S}_{n}(t): \hat{\tau}_{k-1, n}<t \leq \hat{\tau}_{n, n}\right\}
$$

we can do the following: start another simple random walk $S^{1}(t)$ at $\hat{S}_{n}\left(\hat{\tau}_{k-1, n}\right)$ with corresponding stopping times $\tau_{n}^{1}$ and condition the walk so that

$$
S^{1}\left(0, \tau_{n}^{1}\right] \cap \hat{S}\left[0, \hat{\tau}_{k-1, n}\right]=\emptyset ;
$$

then, erase loops from this conditioned path. Let $x \in \partial C^{k}$ and let $\mathcal{B}\left(x, \delta e^{k}\right)$ represent the discrete ball of radius $\delta e^{k}$ about $x$. Proposition 3.3 will show that the probability that the conditioned random walk enters this ball at any time tends to zero as $\delta \rightarrow 0$. Since the loop-erased path is a subpath of the conditioned path, the probability it enters the balls also tends to zero. From this we conclude Proposition 3.2. Before stating and proving this proposition, we review some facts about simple random walks (see [9], Sect. 1.6, for more details). Suppose the simple random walk starts at the origin and $\mathcal{B}$ is a discrete ball of radius $\delta e^{n}$ centered at some $y \in \partial C_{n}$. Let $K_{\delta}$ be sufficiently large such that every discrete ball of radius $\delta e^{n}$ has has at least $\delta^{2} e^{2 n}$ points provided $n \geq K_{\delta}$. If we let $V$ denote the number of visits to $\mathcal{B}$ before leaving the ball of radius $e^{n+1}$, then standard estimates give

$$
\mathbf{E}^{0}[V] \leq c_{1} \delta^{2}
$$

and if $z \in \mathcal{B}$, and $n \geq K_{\delta}$,

$$
\mathbf{E}^{z}[V] \geq c_{2} \delta^{2} \log (1 / \delta)
$$

In particular,

$$
\mathbf{E}^{0}[V \mid V \geq 1] \geq c_{2} \delta^{2} \log (1 / \delta)
$$

and hence

$$
\mathbf{P}^{0}\{V \geq 1\} \leq c[\log (1 / \delta)]^{-1}
$$

Proposition 3.3. There exists a constant $c<\infty$ such that the following is true. Let $K_{\delta}$ be as above, $k<n-1$, and let $\omega=[\omega(0), \ldots, \omega(r)]$ be a random walk path with $\omega(0)=0 ; \omega(t) \in C^{k}, t<r ; \omega(k) \in \partial C^{k}$. Let $S$ be a simple random walk with stopping times $\tau_{m}$ and let $\mathcal{B}$ be a discrete ball of radius $\delta e^{k}$ centered at $y \in \partial C^{k+1}$. Then if $r \geq K_{\delta}$,

$$
\mathbf{P}^{\omega(k)}\left\{S\left(0, \tau_{n}\right] \cap \mathcal{B} \neq \emptyset \mid S\left(0, \tau_{n}\right] \cap \omega=\emptyset\right\} \leq c[\log (1 / \delta)]^{-1} .
$$

Proof. Fix $k_{\delta} \leq k<n-1, \delta>0$, and $\omega, \mathcal{B}$ as in the statement of the theorem (but constants in this proof are independent of all of these). Let

$$
a_{1}=\inf \left\{t: S(t) \in \partial C^{k+(1 / 2)}\right\}, \quad b_{1}=\inf \left\{t: S(t) \in \partial C^{k+2}\right\}
$$

and for $j>1$,

$a_{j}=\inf \left\{t>b_{j-1}: S(t) \in \partial C^{k+(1 / 2)}\right\}, \quad d_{j}=\inf \left\{t>b_{j-1}: S(t) \in \partial C^{k+1}\right\}, \quad b_{j}=\inf \left\{t>a_{j-1}: S(t) \in \partial C^{k+2}\right\}$. 
Standard estimates (see [9], Sect. 1.6 and 3.1) tell us that for $k \geq K_{\delta}, x \in \partial C^{k+2}$,

$$
\begin{aligned}
& \mathbf{P}^{x}\left\{\tau_{k+1}>\tau_{n}\right\} \asymp \mathbf{P}^{x}\left\{\tau_{k+(1 / 2)}>\tau_{n}\right\} \asymp c[n-k]^{-1}, \\
& \mathbf{P}\left\{S\left[a_{j}, b_{j}\right] \cap \mathcal{B} \neq \emptyset \mid S(t), t=0,1, \ldots, a_{j}\right\} \leq c[\log (1 / \delta)]^{-1}, \\
& \mathbf{P}\left\{S\left[b_{j}, a_{j+1}\right] \cap \mathcal{B} \neq \emptyset \mid S(t), t=0,1, \ldots, b_{j}\right\} \leq c[\log (1 / \delta)]^{-1} .
\end{aligned}
$$

Also (see [9], Lem. 2.5.3),

$$
\mathbf{P}\left\{S\left[a_{j}, b_{j}\right] \cap \omega \neq \emptyset \mid S(t), t=0,1, \ldots, a_{j}\right\} \geq c .
$$

Let $j^{*}$ be the largest $j$ such that

$$
\left\{b_{j}<\tau_{n}\right\} .
$$

Then the estimates above show there exists a $u<1$ such that

$$
\mathbf{P}\left\{j^{*}=j ; S\left[0, b_{j}\right] \cap \mathcal{B} \neq \emptyset ; S\left[0, b_{j}\right] \cap \omega=\emptyset\right\} \leq c j u^{j}[\log (1 / \delta)]^{-1}[n-k]^{-1} .
$$

Summing over all $j$ we get for $x \in \partial C^{k+(1 / 2)}$.

$$
\mathbf{P}^{x}\left\{S\left(0, \tau_{n}\right] \cap \mathcal{B} \neq \emptyset ; S\left[0, b_{j}\right] \cap \omega=\emptyset\right\} \leq c[\log (1 / \delta)]^{-1}[n-k]^{-1} .
$$

But it is easy to see for $x \in \partial C^{k+(1 / 2)}$,

$$
\mathbf{P}^{x}\left\{S\left(0, \tau_{n}\right] \cap \mathcal{B}=\emptyset ; S\left[0, b_{j}\right] \cap \omega=\emptyset\right\} \geq c[\log (1 / \delta)]^{-1}[n-k]^{-1} .
$$

(To see this we bound by the probability that

$$
S\left(0, a_{1}\right] \cap \mathcal{B}=\emptyset, S\left[0, b_{j}\right] \cap \omega=\emptyset
$$

times the probability that $d_{2}>\tau_{n}$.) This gives the proposition.

\section{Extremal LENGTH AND ESCAPE PROBABILITIES}

There is a close relationship between escape probabilities for planar Brownian motions and a quantity known as extremal length. In this section we will review some of the basic facts about extremal length, and then use an extremal length estimation to estimate escape probabilities. For more details about extremal length, see $[1,19]$.

Let $\Gamma$ be a collection of piecewise smooth curves $\gamma:[0,1] \rightarrow \mathbb{C}$. Suppose $\rho: \mathbb{C} \rightarrow \mathbb{R}$ is a measurable function with

$$
a(\rho)=\int_{\mathbb{C}} \rho^{2} d x d y<\infty
$$

Let

$$
L(\rho)=L(\rho, \Gamma)=\inf \int_{\gamma} \rho d|z|
$$


where the infimum is over all $\gamma \in \Gamma$. The extremal length $\Delta=\Delta(\Gamma)$ is defined by

$$
\Delta=\sup \frac{L(\rho)^{2}}{a(\rho)}
$$

where the supremum is over all $\rho$ with $a(\rho)<\infty$. The case of greatest interest for us is where $D$ is a bounded domain; $V_{1}, V_{2}$ closed subsets of $\mathbb{C}$; and $\Gamma=\Gamma\left(D, V_{1}, V_{2}\right)$ is the set of all piecewise smooth $\gamma$ with $\gamma(0) \in$ $V_{1}, \gamma(1) \in V_{2}$, and $\gamma(0,1) \subset D$. In this case we write $\Delta=\Delta\left(D, V_{1}, V_{2}\right)$. Extremal length is a conformal invariant, i.e., if $f: D \rightarrow D_{1}$ is a conformal transformation defined up to the boundary then

$$
\Delta\left(f(D), f\left(V_{1} \cap \bar{D}\right), f\left(V_{2} \cap \bar{D}\right)\right)=\Delta\left(D, V_{1}, V_{2}\right)
$$

If $D$ is the rectangle

$$
D=\{x+i y: 0<x<a, 0<y<b\}
$$

and

$$
V_{1}=\{i y: 0 \leq y \leq b\}, \quad V_{2}=\{a+i y: 0 \leq y \leq b\}
$$

then

$$
\Delta\left(D, V_{1}, V_{2}\right)=\frac{a}{b}
$$

with the supremum in (4.1) being taken on by $\rho \equiv 1$. If $D$ is the split annulus

$$
D=\left\{r e^{i \theta}: e^{-n}<r<1,0<\theta<2 \pi\right\}
$$

with

$$
V_{1}=\left\{|z|=e^{-n}\right\}, \quad V_{2}=\{|z|=1\}
$$

then $D$ is conformally equivalent to a rectangle of sides $n$ and $2 \pi$ and hence

$$
\Delta\left(D, V_{1}, V_{2}\right)=\frac{n}{2 \pi}
$$

Suppose $V_{2}$ is a closed set such that every $\gamma:[0,1] \rightarrow \mathbb{C}$ with $\gamma(0) \in V_{1}, \gamma(1) \in V_{3}, \gamma(0,1) \subset D$ satisfies

$$
\gamma(0,1) \cap V_{2} \neq \emptyset
$$

Then ([19], Prop. 9.2)

$$
\Delta\left(D, V_{1}, V_{3}\right) \geq \Delta\left(D, V_{1}, V_{2}\right)+\Delta\left(D, V_{2}, V_{3}\right)
$$

In other words, the extremal length satisfies a reversed triangle inequality. In particular if we let $\mathcal{B}$ denote the unit disk and $\partial_{n}=\left\{|z|=e^{-n}\right\}$, then for any $D \subset \mathcal{B}$,

$$
\Delta\left(D, \partial_{n}, \partial_{0}\right) \geq \sum_{j=1}^{n} \Delta\left(D, \partial_{j}, \partial_{j-1}\right)
$$


Let $E$ be an interval in the unit circle

$$
E=\left\{e^{i \theta}: \theta_{1} \leq \theta \leq \theta_{2}\right\}
$$

with length $l(E)=\theta_{2}-\theta_{1}$. Let $\partial_{r}$ denote the circle of radius $e^{-r}$ as above, and let $\Gamma_{1}=\Gamma_{1}(r, E)=\Gamma\left(\mathcal{B}, \partial_{r}, E\right)$. Let $B_{t}$ denote a standard two dimensional Brownian motion, considered as a complex valued Brownian motion, with stopping times

$$
T_{n}=\inf \left\{t:\left|B_{t}\right|=e^{n}\right\}
$$

By Pfluger's Theorem ( [19], Th. 9.17) for $1 \leq r \leq 5$,

$$
l(E)=\frac{1}{2 \pi} \mathbf{P}^{0}\left\{B\left(T_{0}\right) \in E\right\} \asymp \exp \left\{-\pi \Delta_{1}\right\}
$$

where $\Delta_{1}=\Delta_{1}\left(\mathcal{B}, \partial_{r}, E\right)$. Let $\Gamma_{2}=\Gamma_{2}(r)$ be the collection of curves $\left\{\gamma_{\theta}\right\}$,

$$
\gamma_{\theta}(t)=e^{-i \theta}\left[(1-t) e^{-r-1}+t e^{-r}\right], \quad 0 \leq t \leq 1
$$

Let $\Gamma_{3}=\Gamma_{3}(r, E)$ denote the set of curves obtained by combining any $\gamma_{2} \in \Gamma_{2}$ with any $\gamma_{1} \in \Gamma_{1}$. The way to combine is to start with $\gamma_{2}$; then take a curve in $\partial_{r}$ which goes from $\gamma_{2}(1)$ to $\gamma_{1}(0)$; and then follow $\gamma_{1}$. Of course, we must do a simple reparameterization to get a curve $\gamma:[0,1] \rightarrow \mathbb{C}$. It is not difficult to show that there is a $c$, independent of $r$ and $E$, such that

$$
\Delta\left(\Gamma_{3}\right) \leq \Delta_{1}+c
$$

(In proving this one notes that one can restrict the set of $\rho$ to those that are zero on $\partial_{r}$.) Similarly, let $\Gamma_{4}$ be the collection of curves $\left\{\gamma_{\theta}\right\}$,

$$
\gamma_{\theta}(t)=e^{-i(\theta+2 \pi t)}\left[(1-t) e^{-r-1}+t e^{-r}\right], \quad 0 \leq t \leq 1
$$

and let $\Gamma_{5}$ be the collection of curves obtained by attaching curves from $\Gamma_{4}$ with curves from $\Gamma_{1}$. Again we can show that

$$
\Delta\left(\Gamma_{5}\right) \leq \Delta_{1}+c
$$

By comparison with $\Gamma_{4}$ and $\Gamma_{5}$, we can see that if $h:[0,1] \rightarrow \mathbb{C}$ is any continuous curve with $h(0) \in \partial_{r+1}, h(1) \in$ $\partial_{r}$, then

$$
\Delta(\mathcal{B}, h[0,1], E) \leq \Delta_{1}+c
$$

Now suppose $r \geq 2$ and $h:[0,1] \rightarrow \mathbb{C}$ is a continuous curve without double points with $h(0) \in \partial_{r}, h(1) \in \partial_{0}$, $h[0,1) \subset \mathcal{B}$. Let $D$ be the connected component of $\mathcal{B} \backslash h[0,1]$ whose boundary includes $\partial_{0}$. Let $x \in \partial_{r} \cap D$ (if $\partial_{r} \cap D=\emptyset$, the conclusion we will obtain below is trivial). Let $d$ be the distance from $x$ to $\partial D$, and note that $d \leq 2|x|$; in particular, the closed disk of radius $d$ about $x$ is contained in the disk of radius $e^{-r+2}$ about the origin. Let $f$ be a conformal transformation of $D$ to the unit disk with $f(x)=0$. Let $\delta$ be the smallest number such that $f(\overline{\mathcal{B}}(x, \delta)) \cap \partial_{2} \neq \emptyset$, where $\overline{\mathcal{B}}(x, \delta)$ denotes the closed ball of radius $\delta$ about $x$. Note that $\delta<d$. By conformal invariance of Brownian motion (or equivalently, by conformal invariance of harmonic measure),

$$
\mathbf{P}^{x}\left\{B\left[0, T_{0}\right] \cap h[0,1]=\emptyset\right\}=\mathbf{P}^{0}\left\{B\left(T_{0}\right) \in f\left(\partial_{0}\right)\right\}
$$


By Pfluger's Theorem, the right hand side is comparable to

$$
\exp \left\{-\pi \Delta\left(\mathcal{B}, \partial_{2}, f\left(\partial_{0}\right)\right)\right\}
$$

By (4.3), this is comparable to

$$
\exp \left\{-\pi \Delta\left(\mathcal{B}, f(\overline{\mathcal{B}}(x, \delta)), f\left(\partial_{0}\right)\right)\right\}
$$

which by conformal invariance of extremal distance equals

$$
\exp \left\{-\pi \Delta\left(D, \partial \mathcal{B}(x, \delta), \partial_{0}\right)\right\}
$$

Since $\mathcal{B}(x, \delta) \subset \mathcal{B}\left(0, e^{r-2}\right)$, this last term is smaller than

$$
\exp \left\{-\pi \Delta\left(D, \partial_{r-2}, \partial_{0}\right)\right\} .
$$

Combining all of this we get that there is a constant $c$ such that if $h$ is as above and $x \in \partial_{r}$,

$$
\mathbf{P}^{x}\left\{B\left[0, T_{0}\right] \cap h[0,1]=\emptyset\right\} \leq c \exp \left\{-\pi \Delta\left(D, \partial_{r-2}, \partial_{0}\right)\right\} .
$$

Let $h, D$ be as in the previous paragraph, and let $A$ be the connected component of $D \cap\left\{|z|>e^{-r}\right\}$ that contains the unit circle in its boundary. Assume that $\partial A \cap \partial_{r} \neq \emptyset$. Note that if $\gamma$ is a curve with $\gamma(0) \in \partial_{r}, \gamma(1) \in$ $\partial_{0}, \gamma(0,1) \subset D$, then there exists an $s \geq 0$ such that $\gamma(s) \in \partial_{r}, \gamma(s, 1) \subset A$. Hence,

$$
\Delta\left(D, \partial_{r-2}, \partial_{0}\right)=\Delta\left(A, \partial_{r-2}, \partial_{0}\right) .
$$

It will be convenient if we conformally map this region by a logarithm. In this case $\partial_{r}$ is sent to $U_{r}$ where

$$
U_{j}=\{\Re(z)=-j\} .
$$

We take an $h:[0,1] \rightarrow \mathbb{C}$ satisfying

$$
h(0) \in U_{r}, \quad h(1) \in U_{0}, \quad h(0,1) \subset\{\Re(z)<0\},
$$

and for each integer $k$ we have the curve

$$
h_{k}(t)=h(t)+2 \pi i, \quad 0 \leq t \leq 1 .
$$

Let $G$ be the region bounded by $U_{r}, U_{0}, h\left[\sigma_{r}, 1\right]$ and $h_{1}\left[\sigma_{r}, 1\right]$. The region $A$ is the connected component of $G$ whose boundary intersects $U_{0}$. Note that any curve connecting $U_{r}$ to $U_{0}$ in $G$ has a subpath connecting $U_{r}$ to $U_{0}$ in $A$. Since $A \subset G$,

$$
\operatorname{area}(A) \leq \operatorname{area}(G) \leq 2 \pi r .
$$

If $j$ is a positive integer, let $A_{j}$ denote the connected component of $A \cap\{\Re(z)<-j\}$ whose boundary includes a portion of $U_{r}$, and let $V_{j}=U_{j} \cap \partial A_{j}$. Note that $V_{j}$ is contained in an interval of length at most $2 \pi$, and that every continuous curve from $U_{r}$ to $U_{0}$ staying in $A$ hits $U_{j}$ first in $V_{j}$. Fix some $0<\epsilon<1 / 10$. Suppose we can find $k$ integers $1 \leq j_{1}<\cdots<j_{k} \leq r$ such that the following holds. For each $j \in\left\{j_{1}, \ldots, j_{k}\right\}$, there exists a point $z_{j}$ with

$$
\Re\left(z_{j}\right)=-j+\frac{1}{2},
$$


and such that for each $w_{1}, w_{2} \in \mathcal{B}\left(z_{j}, \epsilon\right)$,

$$
\operatorname{dist}\left(w_{1}, V_{j}+2 \pi l\right)+\operatorname{dist}\left(w_{2}, V_{j-1}+2 \pi l\right) \geq 1,
$$

for every integer $l$. In other words the portion of every path in $A$ from $V_{j}$ to $V_{j-1}$ outside of the $2 \pi i$ integer translates of $\mathcal{B}\left(z_{j}, \epsilon\right)$ must have length at least one. Note that

$$
\operatorname{area}\left[G \backslash \bigcup_{j=1}^{k} \bigcup_{l=-\infty}^{\infty} \mathcal{B}\left(z_{j}+2 \pi l, \epsilon\right)\right] \leq 2 \pi r-k \pi \epsilon^{2} .
$$

If we let $\rho$ be the function that is 1 on

$$
A \backslash\left[\bigcup_{j=1}^{k} \bigcup_{l=-\infty}^{\infty} \mathcal{B}\left(z_{j}+2 \pi l, \epsilon\right)\right]
$$

and zero elsewhere, we have

$$
a(\rho) \leq 2 \pi r-k \pi \epsilon^{2}
$$

and

$$
L(\rho) \geq r
$$

Hence

$$
\Delta\left(A, U_{r}, U_{0}\right) \geq \frac{r^{2}}{2 \pi r-k \pi \epsilon^{2}} \geq \frac{r}{\pi}\left[\frac{1}{2}+\frac{\epsilon^{2} k}{4 r}\right] .
$$

Now fix a $j$ and suppose $s<t$ with $h(s) \in U_{j}, h(t) \in U_{j-1}, h(s, t) \subset\{\Re(z)<j-1\}$. Suppose also that

$$
\Im(h(t)) \geq \Im(h(s))+\delta,
$$

for some $\delta>0$. Note that

$$
V_{j} \subset\{-j+i y: h(s) \leq y \leq h(s)+2 \pi\}, \quad V_{j-1} \subset\{-j+1+i y: h(t) \leq y \leq h(t)+2 \pi\} .
$$

Let

$$
z_{j}=-j+\frac{1}{2}+\left[h(s)+\frac{\delta}{2}\right] i .
$$

Note that if we draw a sufficiently small ball around this point (we leave the high school geometry estimate to the reader), that it satisfies (4.5) for some $\epsilon=\epsilon_{\delta}$. Similarly if $\Im(h(t)) \leq \Im(h(s))-\delta$, a similar fact holds using

$$
z_{j}=-j+\frac{1}{2}+\left[h(s)-\frac{\delta}{2}\right] i
$$

When we transform this argument back to the unit disk and use (4.4), we get the following lemma. (The $\epsilon$ in this lemma corresponds to the $\epsilon^{2} / 4$ above.) 
Lemma 4.1. For every $\delta>0$, there exists an $\epsilon>0$ and a $c<\infty$ such that the following is true. Let $r$ be a positive integer and $h:[0,1] \rightarrow \mathbb{C}$ a continuous function without double points with $h(0) \in \partial_{r}, h(1) \in$ $\partial_{0}, h(0,1) \subset \mathcal{B}$. For each integer $j=1, \ldots, r-2$, let

$$
\nu_{j}=\inf \left\{t: h(t) \in \partial_{j}\right\}, \quad Y_{j}=\left|\arg \left(h\left(\nu_{j}\right)\right)-\arg \left(h\left(\nu_{j-1}\right)\right)\right| .
$$

Let

$$
W=W_{r}(h, \delta)=\sum_{j=1}^{r} I\left\{Y_{j} \geq \delta\right\}
$$

Then if $x \in \partial_{r}$,

$$
\mathbf{P}^{x}\left\{B\left[0, T_{0}\right] \cap h[0,1]=\emptyset\right\} \leq c \exp \left\{-r\left(\frac{1}{2}\right)+\epsilon W\right\}
$$

\section{Strong approximation}

We will need to use a strong approximation of a simple random walk and a Brownian motion in two dimensions. The approximation we will use is derived from the standard Skorohod embedding of a one dimensional simple random walk in a one dimensional Brownian motion. Let $(\Omega, \mathcal{F}, \mathbf{P})$ be a probability space on which are defined a two-dimensional Brownian motion, $B_{t}=B(t)=\left(B_{t}^{1}, B_{t}^{2}\right)$, and a one-dimensional simple random walk, $W_{k}$, that is independent of the Brownian motion. As before, let

$$
T_{n}=\inf \left\{t:\left|B_{t}\right|=e^{n}\right\}
$$

and let

$$
R_{k}=\frac{1}{2}\left(W_{k}+k\right)
$$

Let $S_{k}^{i}$ be the simple random walk derived from $B_{t}^{i}$ from the Skorohod embedding. This is obtained by setting $\eta_{0}^{i}=0$,

$$
\eta_{k+1}^{i}=\inf \left\{t \geq \eta_{k}^{i}:\left|B^{i}(t)-B^{i}\left(\eta_{k}^{i}\right)\right|=1\right\}
$$

and

$$
S_{k}^{i}=B^{i}\left(\eta_{k}^{i}\right)
$$

Since $\mathbf{E}\left[\eta_{1}^{i}\right]=1$, one expects $\eta_{k}^{i}-k$ to be of order $k^{1 / 2}$ and hence $B\left(\eta_{k}^{i}\right)-B(k)$ to be of order $k^{1 / 4}$. Standard techniques make this precise; in particular, since $\eta_{k}^{i}$ has an exponential moment, we can derive exponential estimates on the probabilities. In fact, one can show that there exists $c, \beta$ such that

$$
\mathbf{P}\left\{\sup _{0 \leq s, t \leq e^{9 n / 8},|s-t| \leq e^{5 n / 8}}\left|B_{t}^{i}-B_{s}^{i}\right| \geq e^{3 n / 8}\right\} \leq c \exp \left\{-e^{\beta n}\right\} .
$$

(In this section we let $c, \beta$ be positive constants whose value may change from line to line.) Also,

$$
\mathbf{P}\left\{\sup _{0 \leq k \leq e^{9 n / 8}}\left|\eta_{k}^{i}-k\right| \geq e^{5 n / 8}\right\} \leq c \exp \left\{-e^{\beta n}\right\} .
$$


In particular,

$$
\mathbf{P}\left\{\sup _{0 \leq t \leq e^{9 n / 8}}\left|B_{t}^{i}-S^{i}(\lfloor t\rfloor)\right| \geq e^{3 n / 8}\right\} \leq c \exp \left\{-e^{\beta n}\right\}
$$

If we let

$$
S_{k}=\left(S^{1}\left(R_{k}\right), S^{2}\left(k-R_{k}\right)\right),
$$

it is easy to see that $S_{k}$ is a simple random walk in $\mathbb{Z}^{2}$. Again, standard estimates given

$$
\mathbf{P}\left\{\sup _{0 \leq k \leq e^{9 n / 8}}\left|R_{k}-\frac{k}{2}\right| \geq e^{5 n / 8}\right\} \leq c \exp \left\{-e^{\beta n}\right\}
$$

and hence

$$
\mathbf{P}\left\{\sup _{0 \leq t \leq e^{9 n / 8}}\left|B_{t}-S(\lfloor 2 t\rfloor)\right| \geq c e^{3 n / 8}\right\} \leq c \exp \left\{-e^{\beta n}\right\}
$$

Also note that

$$
\mathbf{P}\left\{T_{n} \geq e^{9 n / 4}\right\} \leq c \exp \left\{-e^{\beta n}\right\} .
$$

We now let $U_{n}$ be the event

$$
U_{n}=\left\{T_{n} \leq e^{9 n / 4} ; \sup _{0 \leq t \leq T_{n}}\left|B_{t}-S(\lfloor 2 t\rfloor)\right| \leq c e^{3 n / 4}\right\} .
$$

This event is measurable with respect to the $\sigma$-algebra generated by

$$
\left\{B_{t}: t \leq T_{n}\right\} \cup\left\{R_{k}, k=0,1,2, \ldots\right\} .
$$

In particular it is independent of the $\sigma$-algebra generated by

$$
\left\{B\left(t+T_{n}\right)-B\left(T_{n}\right): t \geq 0\right\} .
$$

We have sketched the proof of the following. The Brownian motion $B_{t}$ in the lemma has variance parameter $1 / 2$, i.e., $B_{t}=\tilde{B}_{t / 2}$, where $\tilde{B}$ is a standard Brownian motion.

Lemma 5.1. There exists a $c, \beta$ such that a planar Brownian motion with variance parameter $1 / 2$ and a twodimensional simple random walk $S_{k}$ can be defined on the same probability space satisfying the following. For each $n$, there exists an event $E_{n}$ that is independent of

$$
\left\{B\left(t+T_{n}\right)-B\left(T_{n}\right): t \geq 0\right\}
$$

with

$$
\mathbf{P}\left(E_{n}\right) \geq 1-c \exp \left\{-e^{\beta n}\right\}
$$

and such that on the event $E_{n}$,

$$
|B(t)-S(\lfloor t\rfloor)| \leq e^{7 n / 8}, \quad t \leq T_{n-(1 / 2)}
$$


Let

$$
\tau_{n}=\inf \left\{k:\left|S_{k}\right|=e^{n}\right\}
$$

One can see that if $V$ is any event that is measurable with respect to

$$
\left\{S_{k}: 0 \leq k \leq \tau_{n-(1 / 2)}\right\},
$$

then $V \cap E_{n}$ is also independent of the $\sigma$-algebra generated by

$$
\left\{B\left(t+T_{n}\right)-B\left(T_{n}\right): t \geq 0\right\} .
$$

\section{BOUND FOR RANDOM WALKS}

Let $\delta_{0}, \epsilon$ be such that Lemma 4.1 holds (for some constant $c$ ), and choose $\delta>\delta_{0}$. We allow constants $c, c_{1}, c_{2}, \ldots$ in this section to depend on $\delta_{0}, \delta, \epsilon$. The goal of this section is to show that a corresponding result holds for simple random walk for $\delta, \epsilon$. We first use Brownian scaling to make a slight restatement of Lemma 4.1. If $h:[0, \infty) \rightarrow \mathbb{C}$ is a continuous curve without double points with $h(0)=0,|h(t)| \rightarrow \infty, t \rightarrow \infty$, let

$$
\begin{aligned}
\sigma_{n} & =\inf \left\{t:|h(t)|=e^{n}\right\}, \\
Y_{n} & =Y_{n}(h)=\left|\arg \left(h\left(\sigma_{n}\right)\right)-\arg \left(h\left(\sigma_{n+1}\right)\right)\right|, \\
W_{m, n} & =W_{m, n}\left(h, \delta_{0}\right)=\sum_{k=0}^{n-1} I\left\{Y_{m+k} \geq \delta_{0}\right\} .
\end{aligned}
$$

Then Lemma 4.1 immediately implies the following.

Lemma 6.1. There exists a constant $c$ such that for all nonnegative integers $m, n$ and all $h$,

$$
\mathbf{P}^{0}\left\{B\left[T_{m}, T_{m+n}\right] \cap h\left[\sigma_{m}, \sigma_{m+n}\right]=\emptyset\right\} \leq c \exp \left\{-n \frac{1}{2}-\epsilon W_{m, n}\right\} .
$$

Let $\omega:\{0,1,2, \ldots\} \rightarrow \mathbb{Z}^{2}$ be a self-avoiding random walk path with $\omega(0)=0,|\omega(t)| \rightarrow \infty, t \rightarrow \infty$. Associated with $\omega$ is the continuous path $h_{\omega}:[0, \infty) \rightarrow \mathbb{C}$ obtained by linear interpolation, i.e.,

$$
h_{\omega}(t)=\omega(\lfloor t\rfloor)+(t-\lfloor t\rfloor)[\omega(\lfloor t\rfloor+1)-\omega(\lfloor t\rfloor)]
$$

(where we consider $\mathbb{Z}^{2}$ as embedded in $\mathbb{C}$ ). Let

$$
\begin{aligned}
\tilde{\sigma}_{n} & =\inf \left\{t:|\omega(t)| \geq e^{n}\right\}, \\
\tilde{Y}_{n} & =\tilde{Y}_{n}(\omega)=\left|\arg \left(\omega\left(\tilde{\sigma}_{n}\right)\right)-\arg \left(\omega\left(\tilde{\sigma}_{n+1}\right)\right)\right|, \\
\tilde{W}_{m, n} & =\tilde{W}_{m, n}(\omega, \delta)=\sum_{k=0}^{n-1} I\left\{\tilde{Y}_{m+n} \geq \delta\right\} .
\end{aligned}
$$

Note that if $\sigma_{n}$ are the times defined as above for $h=h_{\omega}$,

$$
\sigma_{n} \leq \tilde{\sigma}_{n} \leq \sigma_{n}+1 .
$$

In particular, for all $n$ sufficiently large, if $\tilde{Y}_{n} \geq \delta$, then $Y_{n} \geq \delta_{0}$. Therefore,

$$
W_{m, n}\left(h_{\omega}\right) \geq \tilde{W}_{m, n}(\omega)-c_{1} .
$$

Our goal is to prove the following. 
Lemma 6.2. There exists a constant $c_{2}$ such that for all nonnegative integers $m, n$, and all $\omega$ as above,

$$
\mathbf{P}^{0}\left\{S\left[\tau_{m}, \tau_{m+n}\right] \cap \omega\left[\tilde{\sigma}_{m}, \tilde{\sigma}_{m+n}\right]=\emptyset\right\} \leq c_{2} \exp \left\{-n \frac{1}{2}-\epsilon \tilde{W}_{m, n}\right\}
$$

Let

$$
b_{n}=b_{n}(\delta, \epsilon)=\sup \exp \left\{n \frac{1}{2}+\epsilon \tilde{W}_{m, n}\right\} \mathbf{P}^{0}\left\{S\left[\tau_{m}, \tau_{m+n}\right] \cap \omega\left[\tilde{\sigma}_{m}, \tilde{\sigma}_{m+n}\right]=\emptyset\right\},
$$

where the supremum is over all $\omega$ and all positive integers $m$. Lemma 6.2 is equivalent to saying that the sequence $\left\{b_{n}\right\}$ is bounded. It is obvious that $b_{0}=1$ and since $\tilde{W}_{m, n} \leq n$,

$$
b_{m+n} \leq e^{n \alpha} b_{m}
$$

where $\alpha=(1 / 2)+\epsilon$. If we show that there is a $\beta>0$, and a $c_{3}<\infty$ such that for all $n$,

$$
b_{n} \leq c_{3} \sum_{j=0}^{n-1} e^{-j \beta} b_{j},
$$

then it follows that the $b_{n}$ are bounded (see, for example [12], Lem. 4.5). So to prove Lemma 6.2 it suffices to prove (6.2) for $n \geq 3$.

Let $B$ be a Brownian motion starting at the origin in $\mathbb{C}$ and let $S$ be the corresponding simple random walk derived from the strong approximation as in Lemma 5.1. Let $\omega$ be a self-avoiding random walk path and $h=h_{\omega}$ as above. For $1 \leq k \leq n-1$, let $U_{k}=U_{k}(m, n)$ be the event

$$
U_{k}=\left\{B\left[T_{m+k-1}, T_{m+k}\right] \cap h\left[\sigma_{m}, \sigma_{m+n}\right] \neq \emptyset ; \quad B\left[T_{m+k}, T_{m+n-1}\right] \cap h\left[\sigma_{m}, \sigma_{m+n}\right]=\emptyset\right\} .
$$

Let $U_{0}=U_{0}(m, n)$ be the event

$$
U_{0}=\left\{B\left[T_{m}, T_{m+n-1}\right] \cap h\left[\sigma_{m}, \sigma_{m+n}\right]=\emptyset\right\},
$$

and let $V=V_{m, n}$ be the event

$$
\left\{S\left[\tau_{m}, \tau_{m+n}\right] \cap \omega\left[\tilde{\sigma}_{m}, \tilde{\sigma}_{m+n}\right]=\emptyset\right\}
$$

Note that

$$
V=\bigcup_{k=0}^{n-1}\left(V \cap U_{k}\right) .
$$

To prove (6.2) it suffices to prove that for some positive constants $c, \beta$,

$$
\mathbf{P}\left[V \cap U_{k}\right] \leq c b_{k} e^{-k \beta} \exp \left\{-\frac{1}{2} n-\epsilon \tilde{W}_{m, n}\right\} .
$$

For $k=0,1$, this estimate follows immediately from Lemma 6.1 and (6.1) and hence we will assume $k \geq 2$. Note that

$$
\tilde{W}_{m, m+k-2}+W_{m+k+3, m+n} \geq \tilde{W}_{m, n}-c_{3} .
$$


Fix $k$ and let $E=E_{m+k+2}$ be the event as in Lemma 5.1 and write $\beta_{1}$ for the $\beta$ in that lemma. Note that

$$
\begin{aligned}
\mathbf{P}\left[V \cap U_{k} \cap E^{c}\right] & \leq \mathbf{P}\left[E^{c} ; B\left[T_{m+k+2}, T_{m+n-1}\right] \cap h\left[\sigma_{m+k+2}, \sigma_{m+n}\right]=\emptyset\right] \\
& \leq c \mathbf{P}\left(E^{c}\right) \mathbf{P}\left\{B\left[T_{m+k+3}, T_{m+n-1}\right] \cap h\left[\sigma_{m+k+2}, \sigma_{m+n}\right]=\emptyset\right\} \\
& \leq c \exp \left\{-e^{\beta_{1} k}\right\} \exp \left\{\frac{1}{2}(n-k)-\epsilon W_{m+k+3, m+n-1}\right\} \leq c \exp \left\{-\frac{1}{2} n-\epsilon W_{m, m+n-1}\right\} .
\end{aligned}
$$

The second inequality uses the conditional independence and the Harnack inequality for Brownian motion (harmonic functions), and the last inequality uses the trivial inequality $W_{m, m+k} \leq k$. Note that $W_{m, n+m-1} \geq$ $\tilde{W}_{m, n}-c$.

Let $F=F_{m, k}$ be the event

$$
F=\left\{S\left[\tau_{m}, \tau_{m+k+1}\right] \cap \omega\left[\hat{\sigma}_{m}, \hat{\sigma}_{m+k+1}\right]=\emptyset ; \quad \operatorname{dist}\left(S\left[\tau_{m+k-2}, \tau_{m+k}\right], \omega\left[\hat{\sigma}_{m}, \hat{\sigma}_{m+k}\right]\right) \leq e^{15(m+k) / 16}\right\},
$$

and let

$$
\rho=\rho_{m, k, \omega}=\inf \left\{t \geq \tau_{m+k-2}: \operatorname{dist}\left(S(t), \omega\left[\tau_{m+k-2}, \tau_{m+k}\right]\right) \leq e^{15(m+k) / 16}\right\} .
$$

By the discrete Beurling projection theorem (see [10], Lem. 2.3) for the more general version used here) and the strong Markov property for random walk,

$$
\mathbf{P}\left\{S\left[\rho, \tau_{m+k+1}\right] \cap \omega\left[\hat{\sigma}_{m}, \hat{\sigma}_{m+k+1}\right]=\emptyset \mid \rho \leq \tau_{m+k}\right\} \leq c e^{-k / 32} .
$$

Hence,

$$
\mathbf{P}(F) \leq c b_{k} \exp \left\{-k \frac{1}{2}-\epsilon \tilde{W}_{m, m+k-2}-k \frac{1}{32}\right\} .
$$

However, conditioned on the event $E$, the event $F$ is independent of

$$
\left\{B\left(t+T_{m+k+2}\right)-B\left(T_{m+k+2}\right): t \geq 0\right\} .
$$

Also

$$
V \cap U_{k} \cap E \subset F \cap\left\{B\left[T_{m+k+2}, T_{m+n-1}\right] \cap h\left[\sigma_{m+k+2}, \sigma_{m+n-1}\right]=\emptyset\right\} .
$$

Hence from the strong Markov property for Brownian motion and the Harnack inequality,

$$
\begin{aligned}
\mathbf{P}\left(V \cap U_{k} \cap E\right) & \leq c b_{k} e^{-k / 32} \exp \left\{-n \frac{1}{2}-\epsilon\left(\tilde{W}_{m, m+k-2}+W_{m+k+2, m+n-1}\right)\right\} \\
& \leq c b_{k} e^{-k / 32} \exp \left\{-n \frac{1}{2}-\epsilon \tilde{W}_{m+n}\right\},
\end{aligned}
$$

which gives (6.3) and hence proves Lemma 6.1.

\section{REFERENCES}

[1] Ahlfors L., Conformal Invariance. Topics in Geometric Function Theory. McGraw-Hill (1973).

[2] Billingsley P., Probability and Measure. 2nd ed., John Wiley (1986).

[3] Burdzy K. and Lawler G., Rigorous exponent inequalities for random walks. J. Phys. A. 23 (1990) L23-L28.

[4] Duplantier B., Loop-erased self-avoiding walks in 2D. Physica A 191 (1992) 516-522. 
[5] Fargason C., The percolation dimension of Brownian motion in three dimensions. Ph.D. dissertation, Duke University (1998).

[6] Guttmann A. and Bursill R., Critical exponent for the loop-erased self-avoiding walk by Monte Carlo methods. J. Stat. Phys. 59 (1990) 1-9.

[7] Kenyon R., The asymptotic distribution of the discrete Laplacian (1998) preprint.

[8] Kesten H., Hitting probabilities of random walks on $\mathbb{Z}^{d}$. Stoc. Proc. Appl. 25 (1987) 165-184.

[9] Lawler G., Intersections of Random Walks. Birkhäuser-Boston (1991).

[10] Lawler G., A discrete analogue of a theorem of Makarov. Comb. Prob. Computing 2 (1993) 181-199.

[11] Lawler G., The logarithmic correction for loop-erased walk in four dimensions, Proceedings of the Conference in Honor of Jean-Pierre Kahane (Orsay 1993), special issue of J. Fourier Anal. Appl. (1995) 347-362.

[12] Lawler G., Cut points for simple random walk. Electron. J. Prob. 1 (1996) 13.

[13] Lawler G., Loop-erased random walk, preprint, to appear in volume in honor of Harry Kesten (1998).

[14] Lawler G. and Puckette E., The intersection exponent for simple random walk (1998) preprint.

[15] Madras N. and Slade G., The Self-Avoiding Walk. Birkhäuser-Boston (1993).

[16] Majumdar S.N., Exact fractal dimension of the loop-erased self-avoiding random walk in two dimensions, Phys. Rev. Lett. 68 (1992) 2329-2331.

[17] Pemantle R., Choosing a spanning tree for the integer lattice uniformly. Ann. Prob. 19 (1991) 1559-1574.

[18] Propp J. and Wilson D., How to get a perfectly random sample from a generic Markov chain and generate a random spanning tree of a directed graph. J. Algorithms (to appear).

[19] Pommerenke C., Boundary Behaviour of Conformal Maps, Springer-Verlag (1992).

[20] Werner W., Beurling's projection theorem via one-dimensional Brownian motion. Math. Proc. Cambridge Phil. Soc. 119 (1996) 729-738. 